\title{
Is leptin coming back? A short introduction to the presentations in this symposium session at the 2015 annual meeting of the EASD
}

\author{
Ulf Smith ${ }^{1}$ \\ Received: 11 January 2016 / Accepted: 22 January 2016 / Published online: 4 March 2016 \\ (C) Springer-Verlag Berlin Heidelberg 2016
}

Keywords Adipokine $\cdot$ Diabetes $\cdot$ Leptin $\cdot$ Obesity

\section{Abbreviation \\ HPA Hypothalamic-pituitary-adrenal}

Leptin was identified by Friedman and coworkers in 1994 and this discovery created much enthusiasm and hope that obesity could be treated with this adipose tissue-secreted (adipokine) molecule. However, it was soon found that normal obesity is associated with elevated leptin levels and that exogenous treatment was not a success. These findings then led to the concept of 'leptin resistance' in obesity and raised interest in understanding the molecular mechanisms whereby individuals did not respond to exogenous leptin administration. However, our understanding is still incomplete and requires further work.

Although not the panacea for obesity treatment at large, leptin has been registered as a useful therapy for lipodystrophy and its associated consequences of hyperphagia, ectopic liver fat storage (liver steatosis), liver disease and dyslipidaemia. It has been shown that, in these individuals, treatment with the leptin analogue metreleptin reverses the hyperphagia and reduces the metabolic complications by, for example, lowering fasting glucose levels. It has also successfully been used to treat individuals with genetic leptin deficiencies associated

Ulf Smith

ulf.smith@medic.gu.se

1 The Lundberg Laboratory for Diabetes Research, Department of Molecular and Clinical Medicine, Sahlgrenska Academy, University of Gothenburg, S-41345 Gothenburg, Sweden with morbid obesity (for a review see [1]). However, these conditions are rare and have limited the interest for leptin until recently when it was shown in animal models of type 1 diabetes with insulin deficiency, both streptozotocin-induced diabetes and BB rat models, that treatment with leptin reduced ketogenesis and glucose levels dramatically and without the need for exogenous insulin.

The symposium at the 2015 annual EASD meeting in Stockholm included three experts in the field, who addressed mechanisms for these insulin-independent effects of leptin, as well as how to overcome the leptin resistance in obesity and type 2 diabetes, each of whom has written a short review for this issue [2-4]. A key outstanding question is whether these interesting findings in animal models can be translated to human type 1 diabetes. Clinical studies are ongoing and these results are awaited with great interest.

The first presentation of the symposium was given by Gerald Shulman (New Haven, USA), who summarised what we know about the effects of leptin in individuals with lipodystrophy and the potential mechanisms for the insulinindependent effects seen in animal models. Recent studies have clearly shown that reduced glucagon cannot alone explain all these insulin-independent effects, although it is of major importance for the reduced ketogenesis seen. It was also emphasised that central effects are critically important and that leptin is able to lower corticosteroid levels through effects in the hypothalamic-pituitary-adrenal axis (HPA). Lowering corticosteroid levels reduces adipose tissue lipolysis and substrate-driven hepatic gluconeogenesis.

The mechanisms for this effect of leptin were further elaborated on by Thomas Meek (Seattle, USA), who also emphasised the importance of central effects but claimed that no single effect, such as reduced HPA activation and lower corticosteroid levels, can explain the insulin-independent effects of leptin. He concluded that leptin is a pleiotropic 
factor and that central effects are pivotal and involve (though not exclusively) the HPA axis.

Christoffer Clemmensen (Neuherberg, Germany) summarised what we know about leptin resistance and how it could be overcome. Several potential mechanisms were discussed, and it was explained that in animal models co-therapy with different agents including metformin, glucagon-like peptide (GLP)1 and amylin can at least partly overcome this resistance. However, whether these findings can be translated into an effective therapy in obese human individuals is still not clear. The interesting developments by the Matthias Tschöp laboratory with dual receptor co-agonists may become a novel way of treating obesity and other human diseases.

The three reviews published in this issue describe the current state-of-the-art of our knowledge about leptin, its mechanisms and its potential future usefulness in type 1 diabetes but also emphasise that much work remains to be done.
Duality of interest The author declares that there is no duality of interest associated with this manuscript.

Contribution statement The author is the sole contributor to this paper.

\section{References}

1. Farooqi IS, O'Rahilly S (2014) 20 years of leptin: human disorders of leptin action. J Endocrinol 223:T63-T70

2. Perry RJ, Petersen KF, Shulman GI (2016) Pleotropic effects of leptin to reverse insulin resistance in lipodystrophy and fasting hyperglycemia in rodent models of type 1 diabetes. Diabetologia. doi:10.1007/s00125-016-3909-4

3. Meek TH, Morton GJ (2016) The role of leptin in diabetes: metabolic effects. Diabetologia. doi:10.1007/s00125-016-3898-3

4. Quarta C, Sánchez-Garrido MA, Tschöp MH, Clemmensen C (2016) Renaissance of leptin for obesity therapy. Diabetologia. doi:10.1007/ s00125-016-3906-7 